\title{
Inkjet Printing of Patterned Ultra-Slippery Surfaces for Planar Droplet Manipulation
}

\author{
Shiquan Ling ${ }^{a}$, Yong Luo ${ }^{a}$, Lin Luan ${ }^{b}$, Zhiwei Wang ${ }^{a,{ }^{*}}$, and Tianzhun Wu ${ }^{a,{ }^{*}}$ \\ ${ }^{a}$ Shenzhen Institutes of Advanced Technology, Chinese Academy of Sciences,
} Shenzhen, 518055, China. Tel: +86-755-8639-2339

${ }^{\mathrm{b}}$ Kuang-Chi Institute of Advanced Technology, Shenzhen 518057, China.

* Corresponding author. Email: zw.wang@siat.ac.cn, tz.wu@siat.ac.cn 


\begin{abstract}
We propose a facile, inexpensive and scalable inkjet-printing method to fabricate patterned lubricant-infused surfaces as the ultra-slippery platform for surface microfluidics, and demonstrate typical planar droplet manipulations. Micrometer patterns were printed on scotch tapes by a home-use inkjet printer to form a template with patterned adhesion contrast. Closely packed monolayer of microbeads were assembled driven by the water surface tension and selectively adhered on the unprinted tape. Then poly(dimethylsiloxane) (PDMS) was cast against the microbead template to form a bowl-like surface, and ultra-slippery PDMS surface was achieved after spin coating lubricant oil. Its interface characterization, sliding performances and long-time stability which are critical for surface microfluidic applications were evaluated and analyzed. Finally the patterned ultra-slippery PDMS were employed as the surface microfluidic platform, and droplet transportation, mixing, trapping and releasing functions have been enabled by very simple and facile driving methods such as pneumatic and gravity driving, which pave a new way for potential flexible and low-cost applications of surface microfluidics.
\end{abstract}

\title{
Keywords
}

ultra-slippery surfaces, microfabrication, contact angle hysteresis, droplet manipulation, surface microfluidics 


\section{Introduction}

Surface microfluidics, or planar microfluidics, has recently attracted intensive research interests due to their unique advantages compared with traditional channel-based microfluidics, featured with facile fabrication, direct access and easy integration [1-3]. For the surface microfluidic platform, various droplet manipulation methods have been widely used on typically $\mathrm{Si}$ or glass substrate, such as electrowetting on dielectric (EWOD) [4], optoelectrowetting (OEW) [5], liquid dielectrophoresis (L-DEP) [6], surface acoustic wave (SAW) [7], magnetic [8] or thermocapillary actuation [9]. However, for all of the driving methods the strong contact angle hysteresis $(\mathrm{CAH})$ lead to the higher driving energy and has been recognized as the common bottleneck for droplet manipulation on surfaces. Although superhydrophobic or superlyophobic surfaces $[10,11]$ with micro-/nano- surface structures to minimize actual liquid-solid contact have been proposed to largely reduce $\mathrm{CAH}$ and move droplet effectively, the risk of wetting transition from the Cassie-Baxter state to the Wenzel state under pressure hindered their application in surface microfluidics [12-14], not to mention that the micro/nano structures which provide robust superhydrophobic or superlyophobic performance are typically difficult for fabrication.

Since 2011, Aizenberg's group [15, 16] has developed bio-inspired self-repairing ultra-slippery surfaces named SLIPS (Slippery Liquid Infused Porous Surfaces) by locking lubricant oils in porous or micro array of solid substrates, which have been intensively studied for the purpose of anti-dirt, anti-ice, anti-frost, and anti-biofouling [17-19]. Due to the incompressible and defect-free liquid surface of lubricant oil on SLIPS, they can greatly reduce $\mathrm{CAH}$ and provide extreme pressure stability, which can be a good candidate for 
surface microfluidics. However, so far the application of SLIPS in surface microfluidics remains rarely explored due to several limitations [20-22]. Firstly, uniform and well-controlled micro/nano surfaces which can lock lubricants efficiently are highly preferred for SLIPS [15, 23, 24], however, most SLIPS reported in literature were based on bulky and porous materials [25-28], and it is still difficult to control structure uniformity and hence slippery performances on a large area.

Secondly, special structure patterns are usually necessary in surface microfluidics to precisely guide droplets along a specific trace and achieve more complicated operations. Therefore additional processes are required to obtain specific pattern on SLIPS, yet they are difficult to be applied in existing fabrication methods for SLIPS [29-31].

Thirdly, sufficient oil is required on SLIPS to completely cover the structured solid surfaces to avoid pinning effect $[24,32]$, nevertheless excess oil can exhibit good ultra-slippery performance temporarily and the excess oil will gradually drain away under gravity, resulting in the degradation of slippery performance. Hence the characterization of the ultra-slippery performance of SLIPS should be unified.

Herein we propose a facile, inexpensive and scalable approach to fabricate patterned ultra-slippery poly(dimethylsiloxane) (PDMS) surfaces using the simple yet effective self-assembly polystyrene (PS) microbeads on scotch tapes with the aid of an inkjet printer, and systematically evaluated their ultra-slippery performances and long-term stability for surface microfluidic applications. Typical droplet manipulations such as droplet transportation, mixing, trapping and releasing were demonstrated on the as-prepared ultra-slippery platform using facile gravity and pneumatic driving. Such patternable 
ultra-slippery surfaces provide a new platform for surface microfluidics, and enables simple, inexpensive and programmable driving methods.

\section{Materials and methods}

\section{Materials}

A common scotch tape (Scotch 600, 3M, USA), a commercial ink-jet printer (LBP6018, Canon, Japan) with the original cartridge (Canon CRG-925), PS microbeads (5 $\mu \mathrm{m}, 20 \mu \mathrm{m}, 50$ $\mu \mathrm{m}$ and $100 \mu \mathrm{m}, \mathrm{KB}$ sphere, China) and PDMS (Sylgard 184, base: curing agent $=10: 1$, Dow Corning, USA) were used for fabricating and patterning ultra-slippery surfaces. Methyltrichlorosilane ( 99 wt\%, Sigma Aldrich, USA) was used for low-surface-energy treatment of PDMS for easy demolding. Toluene ( $99.8 \mathrm{wt} \%$, Sigma Aldrich, USA) was used to dissolve the residual PS microbeads, and phenyl silicone oils $(100 \mathrm{cP}, 300 \mathrm{cP}, 500 \mathrm{cP}$ and $1000 \mathrm{cP}$, Sinopharm, China) were used as the lubricant.

\section{Methods}

Firstly, as-required micro pattern was drawn in a computer and printed on a scotch tape using the inkjet printer in order to selectively block adhesion area with the resolution better than $500 \mu \mathrm{m}$ (Figure 1a). Then the tape was immersed with an aqueous solution containing homogeneous PS microbeads (Figure 1b). Multi-layer microbeads were closely packed on the whole scotch tape driven by water surface tension after evaporation or manual extraction of water (Figure 1c). Because the adhesive force of the tape is stronger than adhesion between PS beads to PS beads and ink to PS beads, by applying a moderate pressure to squeeze the 
microbeads on the tape and shaking off redundant microbeads without being stuck to the tape, compactly packed monolayer PS microbeads were obtained, as the negative template for the required pattern (Figure 1d). After the low-surface-tension modification of PS with methyltrichlorosilane treatment, PDMS precursor was casted against the template and demolded following the standard soft lithography method [33]. Residual microbeads stuck on PDMS were then dissolved by toluene washing (Figure 1e). Finally, phenyl silicone oil was spin-coated on the bowl-like PDMS microstructures (Figure 1f).

For systematic comparison, different sizes of PS microbeads, as well as oils with different viscosities $(100 \mathrm{cP}, 300 \mathrm{cP}, 500 \mathrm{cP}$ and $1000 \mathrm{cP})$, were used to evaluate the ultra-slippery performance and durability on PDMS. First, the as-prepared samples were vertically placed for different time intervals and their residual oil weights were measured for quantitative analysis of the oil film thickness. Second, the critical sliding angles of water on these samples were measured by using the contact angle profiler (DSA25, KRUSS, Germany). Finally, the patterned ultra-slippery PDMS surface was used as a surface microfluidic platform to demonstrate typical droplet manipulations, such as droplet transportation, mixing, trapping and releasing using two simple driving methods, gravity and pneumatic driving. The tilting stage used for gravity-based driving was controlled by the home-made open-source hardware (Arduino Uno R3, Seeed, China). Minipumps (KPV36A, KOGE, China) were used for pneumatic driving.

\section{Results and discussions}

\subsection{Characterization of the oil-solid interface}


Figure 2 shows the optical microscopic and scanning electronic microscopic (SEM, NanoSEM 450, Nova, USA) pictures of the assembled PS microbeads with different sizes, as well as their negative replication on PDMS. Closely-packed and uniform microstructures have been readily achieved.

For the long-term stability test, PDMS samples with different porous sizes $(5 \mu \mathrm{m}, 20 \mu \mathrm{m}$, $50 \mu \mathrm{m}$ and $100 \mu \mathrm{m})$ and phenyl silicone oils with different viscosities $(100 \mathrm{cP}, 300 \mathrm{cP}, 500 \mathrm{cP}$ and $1000 \mathrm{cP}$ ) were tested (see Figure S1 ESI), yet only data for $100 \mathrm{cP}$ and $1000 \mathrm{cP}$ were shown in Figure 3 for clarity. As shown in Figures 3a-b and Figure S2 (ESI), in the initial 8 hours, oil residue on PDMS decreased rapidly mainly due to the flowing off of oil, and after that the decrement was much slower, indicating that oil was effectively locked by microstructures, and evaporation should play the dominant role. Therefore all experiments for sliding performance characterization were conducted after vertically placing the samples for 8 hours, to eliminate the rapid reduction of oil thickness. These results also showed that lower oil viscosity and smaller porous size led to less oil residual, thinner lubricate layer and better sliding performance. For example, when oil viscosity is $100 \mathrm{cP}$, the oil residual for samples with $5 \mu \mathrm{m}$ size reaches a stable level of $\sim 25.6 \mu \mathrm{g} / \mathrm{mm}^{2}$, while for samples with $100 \mu \mathrm{m}$ porous size, it is about $40.1 \mu \mathrm{g} / \mathrm{mm}^{2}$ (Figure 3a). Similar trend occurred for samples with oil viscosity $1000 \mathrm{cP}$, and the residual oil significantly increased to $36.6 \mu \mathrm{g} / \mathrm{mm}^{2}$ (for $5 \mu \mathrm{m}$ size) and $51.8 \mu \mathrm{g} / \mathrm{mm}^{2}$ (for $100 \mu \mathrm{m}$ size).

We measured the critical sliding angle (CSA) with different porous sizes, oil viscosities and water droplet volumes. As shown in Figures 3a-b, CSA decreased with smaller porous size, higher oil viscosity and larger water droplet (see Figure S1 in the ESI), therefore the 
optimal configuration in our experiments for best sliding performance $\left(\mathrm{CSA} \sim 3^{\circ}\right.$ ) is $5 \mu \mathrm{m}$ porous size, oil viscosity of $1000 \mathrm{cP}$.

Another simple method to evaluate the oil residual thickness is the transparency test for PDMS samples, considering that oil-solid state can affect the transparency as well as the slippery property [34]. As shown in Figure 3c, transparency became worse for larger porous sizes, and the sample with $5 \mu \mathrm{m}$ porous size demonstrated the best transparency. Therefore, it is concluded that minimizing the porous size yields better oil locking ability for long-time use despite less residual oil, which is consistent with previous report [34]. This may be explained by the scaling effect of surface to volume ratio $(\mathrm{S} / \mathrm{V})$. With reducing porous size, $\mathrm{S} / \mathrm{V}$ of oil increases and the oil-solid adhesion per area increases, which is preferred for droplet thermodynamics, therefore enhances the oil locking effect.

\subsection{Analysis of water-oil interface}

The droplet status on SLIPS is essential for droplet manipulation. Several studies showed that droplets can move easily only when droplet floated on the lubricant [24, 32, 35]. D. Quéré proposed a criterion to judge whether a water droplet floats on oil or contacts with solid [36]:

$$
\Delta \gamma=\gamma_{\mathrm{oa}} \cos \theta_{\mathrm{os}}-\gamma_{\mathrm{wa}} \cos \theta_{\mathrm{ws}}-\gamma_{\mathrm{wo}}>0
$$

Where $\gamma$ represents surface tension, $\theta$ represents intrinsic contact angle, and subscript $\mathrm{w}, \mathrm{o}, \mathrm{a}$, s represents water, oil, air and solid, respectively. When $\Delta y>0$, water is floating on oil. In our experiments using silicon oil with $1000 \mathrm{cP}, \gamma_{\mathrm{wo}}$ is measured as $16.23 \pm 0.64 \mathrm{mN} / \mathrm{m}$, $\gamma_{\mathrm{wa}}=69.34 \pm 0.62 \mathrm{mN} / \mathrm{m}, \quad \gamma_{\mathrm{oa}}=21.31 \pm 0.10 \mathrm{mN} / \mathrm{m}$ while $\theta_{\mathrm{ws}}$ and $\theta_{\mathrm{os}}$ are calculated as $(106.36 \pm 2.06)^{\circ}$ and $(13.96 \pm 1.62)^{\circ}$, respectively (see Table S3 in the ESI for details). 
According to Eq. 1, $\Delta \gamma=23.98 \mathrm{mN} / \mathrm{m}$. Therefore, a water droplet should be floated on the silicone oil without contacting with solid, and therefore allows good sliding performances.

Furthermore, there are two possible states in the oil-water system: water totally cloaked by oil or water floated on oil. For surface microfluidic applications, the former is highly preferred since the existence of oil film can largely prevent water evaporation. The judgment is based on the calculation of spreading coefficient $S_{\text {ow }}$ between oil and water, expressed as [32]:

$$
S_{\text {ow }} \equiv \gamma_{\text {wa }}-\gamma_{\text {wo }}-\gamma_{\text {oa }}>0
$$

In our configuration $S_{\mathrm{ow}}=31.80 \mathrm{mN} / \mathrm{m}>0$, so the silicone oil will cloak the water droplet to minimize the surface energy of the whole system. Therefore such SLIPS platform is very advantageous for aqueous droplet manipulation in surface microfluidics by reducing water evaporation.

We also examined the internal flow pattern inside a droplet. According to Smith et al., the criterion for analyzing the internal flow status is written as [32]:

$$
v / v_{\mathrm{i}} \propto\left(1+\frac{\mu_{\mathrm{o}}}{\mu_{\mathrm{w}}} \frac{h}{t}\right)
$$

where $v$ is the droplet velocity, $v_{\mathrm{i}}$ is the velocity of oil-water interface, $\mu_{\mathrm{o}}$ and $\mu_{\mathrm{w}}$ respectively represents the dynamic viscosity of oil and water. $h$ is the height of the droplet mass center above the solid surface, and $t$ is the oil film thickness. In our case $h \gg t$ so that $\left(1+\mu_{0} / \mu_{\mathrm{w}} h / t\right) \gg 1$ and $v / v_{\mathrm{i}} \gg 1$, which suggests a water droplet mainly rolls on SLIPS (see Figure 4), which is beneficial to accelerate droplet mixing.

\subsection{Demonstration of droplet manipulation}


Based on the above analysis, we fabricated patterned ultra-slippery PDMS (5 $\mu \mathrm{m}$ porous size, $1000 \mathrm{cP}$ silicone oil) to demonstrate their application as a surface microfluidic platform (Figure 5). In Figure 5a, a Y-shape sample and a zigzag-shape sample were obtained by inkjet printing (illustrated in Figure 1a). Figure 5c shows a large-area ultra-slippery PDMS and Figure 5d shows an ultra-slippery ring-shape PDMS with a sliding water droplet on it.

Such surfaces have been used to demonstrate typical droplet manipulations. Figures 6a-c show that droplets were transported, mixed and split in an X-shape ultra-slippery shallow channel defined by the tapes, and Figures 6d-e show the batch movement of a droplet array. Three water droplets (with yellow dye) were dispensed into separate branches, and moved together towards the opposite side by manually tilting the substrate. Programmable driving by gravity was also demonstrated using a simple rotary stage. Figure $6 \mathrm{~g}-1$ show a PDMS surface with an ultra-slippery zigzag path fixed on a tilt rotary stage. The rotation controlled the gravity component and thus the transport direction. 9 traps were designed to store sequential droplets to form a droplet array (Figure 6g-j), and then droplets were simultaneously released (Figure 6k, 1). Note that the blue droplet on the normal PDMS surface did not move in any cases for comparison. These exemplified the potential applications of ultra-slippery surfaces as the novel surface microfluidic platform for facile and low-resistance droplet manipulation based on gravity.

Droplets can also be easily manipulated through other simple driving methods such as blowing. Figure 7 shows that droplets were transported along the designed $\mathrm{X}$-shape slippery paths and mixed at the appointed spot with fast transport speed up to $20 \mathrm{~mm} / \mathrm{s}$ in a well-defined time sequence, by using low-pressure air blow generated by mini-pumps (see the 
video in ESI S4). Such pneumatic driving should be readily programmed to enable the fully automatic control, direct access of individual droplets with low-cost and easy use (see the concept video in ESI S5). Therefore, compared with the traditional driving methods in surface microfluidics, such as electrowetting, magnetic actuation, thermo-capillarity and SAW, non-electrical gravity driving and pneumatic driving methods on SLIPS provide low-cost, high-speed and great flexibility for manipulating individual as well as batch droplets, which may inspire many practical applications of surface microfluidics.

\section{Conclusions}

In summary, a facile, inexpensive and scalable method using inkjet printer to define the ultra-slippery pattern is proposed to fabricate the bowl-like and lubricant-infused ultra-slippery surfaces as the novel platform for droplet manipulation in surface microfluidics. Common scotch tapes with inkjet-printed patterns were used to selectively stick monolayer PS microbeads onto tapes. We analyzed the lubricant locking ability of each substrate for long time use, and examined effects of microbead sizes, viscosity of lubricant oils and droplet sizes. We found that smaller porous sizes and higher oil viscosity yield better oil locking effect by increasing the oil-solid adhesion, and therefore led to smaller CSA.

We also analyzed the droplet status on the ultra-slippery surfaces. In our experiments we found droplets are floated on the silicone oil, cloaked by oil and will be rolling during transportation, which endows superiority for droplet manipulation to reduce motion resistance and water evaporation, and accelerate mixing.

Finally, we demonstrate the typical droplet manipulation such as droplet transportation, mixing, trapping and releasing on the patterned ultra-slippery PDMS surfaces using gravity as 
well as pneumatic driving. Both methods are simple, non-electrical and programmable, and hence suitable for biological/chemical reactions and cell manipulation. These achievements shed new light to surface microfluidics by using patterned lubricant-infused surfaces as the low-cost and versatile platform.

\section{Acknowledgements}

This work is supported by National Science Foundation of China (No.51475451, 51406221), Guangdong Innovative and Entrepreneurial Research Team Program (No.2013S046), Guangdong Natural Science Foundation (2014A030310138, 2015A030306021), Major Program of Guangdong Science and Technology Project (2015B020227002), Shenzhen Peacock Plan, Shenzhen Science and Technology Research Program (JCYJ20140417113430581).

\section{Figure captions}

Figure 1 Schematic illustration of the microfabrication strategy. The scotch tape was printed to obtain the patterned adhesive region(a), then it was fixed on a glass slide and immersed into water (b).PS microbeads were added and water was removed by evaporation or manual extraction (c) to enable closely packed microbead assembly. After removing floating microbeads, a monolayer PS sticking to the tape was obtained (d). PDMS was cast against the PS microbead template (e), and residual microbeads on PDMS were dissolved by toluene. Eventually ultra-slippery PDMS surface was achieved after spin coating lubricant oil (f).

Figure 2 Microscopic pictures of monolayer PS microbeads (5-100 $\mu \mathrm{m})$. In(a)-(d), left side shows PS microbeads on the adhesive tape, and right side shows SEM images of the reverse porous microstructures on PDMS. 
Figure 3 Characterization of stability, sliding performance and transparency of ultra-slippery PDMS. In (1-b), lubricating oil was found to flow down in the initial 8 hours so that residue oil reduced rapidly, and after that oil evaporation played a main role and the residue oil reduced slowly. Samples with smaller porous size and less viscous oil have less oil residue. The critical sliding angle (CSA) for different porous sizes decreases with larger droplet and higher viscosity of silicone oil. In (c), the declining transparency of samples also reflects the decreasing oil thickness. See ESI Figure S1-S2 for more data.

Figure 4 Illustration of a water droplet rolls on the ultra-slippery surface and is cloaked by thin oil film.

Figure 5 As-fabricated ultra-slippery PDMS samples. (a-b) a Y-shape and a zigzag-shape samples obtained by inkjet printing (Figure 1). (c) A large-area (11 cm x $7 \mathrm{~cm}$ ) sample. (d) A ring-shape sample with a water droplet.

Figure $6 \mathrm{X}$-shape and zigzag-shape slippery channels for typical droplet operations. (a-c) Droplets transportation, mixing and splitting in the as-fabricated slippery X-shape channel. (d-f) Droplets dispensed to different branches and the droplet array was simultaneously transported. Sample with a zigzag-shape slippery path was fixed on a tilt rotary stage. By controlling the transport direction and speed, droplet array with 9 trapping slots could store sequential droplets $(g-j)$, and then they were simultaneously released $(\mathrm{k}-\mathrm{l})$. Blue droplets on the normal PDMS surface (d-i) did not move in any cases for comparison.

Figure 7 Pneumatic driven droplet mixing along an X-Shape ultra slippery path. A sample with an X-Shape slippery path was used to mix droplets at the appointed location by controlling the sequence of droplet mixing. Low-pressure air blow was used to push forward droplets with facile and precise control. 


\section{References}

[1] B. Zhao, J.S. Moore, D.J. Beebe, Surface-directed liquid flow inside microchannels, Science 291(2001) 1023-1026.

[2] S.Y. Teh, R. Lin, L.H. Hung, A.P. Lee, Droplet microfluidics, Lab Chip 8(2008) 198-220.

[3] A. Ghosh, R. Ganguly, T.M. Schutzius, C.M. Megaridis, Wettability patterning for high-rate, pumpless fluid transport on open, non-planar microfluidic platforms, Lab Chip 14(2014) 1538-1550.

[4] S.K. Cho, H.J. Moon, C.J. Kim, Creating, transporting, cutting, and merging liquid droplets by electrowetting-based actuation for digital microfluidic circuits, J. Microelectromech. Syst. 12(2003) 70-80.

[5] K.T. Kotz, K.A. Noble, G.W. Faris, Optical microfluidics, Appl. Phys. Lett. 85(2004) 2658-2660.

[6] T.Z. Wu, Y. Suzuki, Liquid dielectrophoresis on electret: a novel approach towards CMOS-driven digital microfludics, J. Adhes. Sci. Technol. 26(2012) 2025-2045.

[7] Z. Guttenberg, H. Muller, H. Habermuller, A. Geisbauer, J. Pipper, J. Felbel, et al., Planar chip device for PCR and hybridization with surface acoustic wave pump, Lab Chip 5(2005) 308-317.

[8] R.S. Sista, A.E. Eckhardt, V. Srinivasan, M.G. Pollack, S. Palanki, V.K. Pamula, Heterogeneous immunoassays using magnetic beads on a digital microfluidic platform, Lab Chip 8(2008) 2188-2196.

[9] J.Z. Chen, A.A. Darhuber, S.M. Troian, S. Wagner, Capacitive sensing of droplets for microfluidic devices based on thermocapillary actuation, Lab Chip 4(2004) 473-480.

[10] A. Accardo, F. Mecarini, M. Leoncini, F. Brandi, E. Di Cola, M. Burghammer, et al., Fast, active droplet interaction: coalescence and reactive mixing controlled by electrowetting on a superhydrophobic surface, Lab Chip 13(2013) 332-335.

[11] M. Jonsson-Niedziolka, F. Lapierre, Y. Coffinier, S.J. Parry, F. Zoueshtiagh, T. Foat, et al., EWOD driven cleaning of bioparticles on hydrophobic and superhydrophobic surfaces, Lab Chip 11(2011) 490-496.

[12] T. Wu, Y. Suzuki, Engineering superlyophobic surfaces as the microfluidic platform for droplet manipulation, Lab Chip 11(2011) 3121-3129.

[13] Z. Wang, T. Wu, Modeling pressure stability and contact-angle hysteresis of superlyophobic surfaces based on local contact line, J. Phys. Chem. C 119(2015) 12916-12922.

[14] K. Y. Song, K. Morimoto, Y. C. Chen, Y. Suzuki, Electrostatic instability of liquid droplets on MEMS-based pillared surfaces, Sensor. Actuat. B-Chem. 225(2016) 492-497.

[15] T.S. Wong, S.H. Kang, S.K. Tang, E.J. Smythe, B.D. Hatton, A. Grinthal, et al., Bioinspired self-repairing slippery surfaces with pressure-stable omniphobicity, Nature 477(2011) 443-447.

[16] N. Vogel, R.A. Belisle, B. Hatton, T.S. Wong, J. Aizenberg, Transparency and damage tolerance of patternable omniphobic lubricated surfaces based on inverse colloidal 
monolayers, Nat. Commun. 4(2013) 2167.

[17] A.K. Epstein, T.S. Wong, R.A. Belisle, E.M. Boggs, J. Aizenberg, Liquid-infused structured surfaces with exceptional anti-biofouling performance, Proc. Natl. Acad. Sci. USA 109(2012) 13182-13187.

[18] D.C. Leslie, A. Waterhouse, J.B. Berthet, T.M. Valentin, A.L. Watters, A. Jain, et al., A bioinspired omniphobic surface coating on medical devices prevents thrombosis and biofouling, Nat. Biotechnol. 32(2014) 1134-1140.

[19] P. Kim, T.S. Wong, J. Alvarenga, M.J. Kreder, W.E. Adorno-Martinez, J. Aizenberg, Liquid-infused nanostructured surfaces with extreme anti-ice and anti-frost performance, ACS Nano 6(2012) 6569-6577.

[20] L. Chen, A. Geissler, E. Bonaccurso, K. Zhang, Transparent slippery surfaces made with sustainable porous cellulose lauroyl ester films, ACS Appl. Mater. Interfaces 6(2014) 6969-6976.

[21] X. Yao, Y. Hu, A. Grinthal, T.S. Wong, L. Mahadevan, J. Aizenberg, Adaptive fluid-infused porous films with tunable transparency and wettability, Nat. Mater. 12(2013) 529-534.

[22] C. Hao, Y. Liu, X. Chen, Y. He, Q. Li, K.Y. Li, et al., Electrowetting on liquid-infused film (EWOLF): complete reversibility and controlled droplet oscillation suppression for fast optical imaging, Sci. Rep. 4(2014) 6846.

[23] D. Daniel, M.N. Mankin, R.A. Belisle, T.S. Wong, J. Aizenberg, Lubricant-infused micro/nano-structured surfaces with tunable dynamic omniphobicity at high temperatures, Appl. Phys. Lett. 102(2013) 231603.

[24] X. Dai, B.B. Stogin, S. Yang, T.S. Wong, Slippery Wenzel State, ACS Nano 9(2015) 9260-9267.

[25] J.T. Simpson, S.R. Hunter, T. Aytug, Superhydrophobic materials and coatings: a review, Rep. Prog. Phys. 78(2015) 086501.

[26] S. Wang, K. Liu, X. Yao, L. Jiang, Bioinspired surfaces with superwettability: new insight on theory, design, and applications, Chem. Rev. 115(2015) 8230-8293.

[27] A. Grinthal, J. Aizenberg, Mobile Interfaces: Liquids as a perfect structural material for multifunctional, antifouling surfaces, Chem. Mater. 26(2014) 698-708.

[28] A.C. Glavan, R.V. Martinez, A.B. Subramaniam, H.J. Yoon, R.M.D. Nunes, H. Lange, et al., Omniphobic "R-F Paper" produced by silanization of paper with fluoroalkyltrichlorosilanes, Adv. Funct. Mater. 24(2014) 60-70.

[29] X. Yao, S.S. Dunn, P. Kim, M. Duffy, J. Alvarenga, J. Aizenberg, Fluorogel elastomers with tunable transparency, elasticity, shape-memory, and antifouling properties, Angew. Chem. Int. Edit. 53(2014) 4418-4422.

[30] J. Yang, H.J. Song, H.Y. Ji, B.B. Chen, Slippery lubricant-infused textured aluminum surfaces, J. Adhes. Sci. Technol. 28(2014) 1949-1957.

[31] S. Sunny, N. Vogel, C. Howell, T.L. Vu, J. Aizenberg, Lubricant-infused nanoparticulate coatings assembled by layer-by-layer deposition, Adv. Funct. Mater. 24(2014) 6658-6667.

[32] J.D. Smith, R. Dhiman, S. Anand, E. Reza-Garduno, R.E. Cohen, G.H. McKinley, et al., Droplet mobility on lubricant-impregnated surfaces, Soft Matter 9(2013) 1772-1780.

[33] Y.N. Xia, G.M. Whitesides, Soft lithography, Angew. Chem. Int. Edit. 37(1998) 
$550-575$.

[34] X. Yao, Y. Hu, A. Grinthal, T.S. Wong, L. Mahadevan, J. Aizenberg, Adaptive fluid-infused porous films with tunable transparency and wettability, Nat. Mater. 12(2013) 529-534.

[35] H. Liu, P. Zhang, M. Liu, S. Wang, L. Jiang, Organogel-based thin films for self-cleaning on various surfaces, Adv. Mater. 25(2013) 4477-4481.

[36] A. Lafuma, D. Quere, Slippery pre-suffused surfaces, EPL-Europhys. Lett. 96(2011) 56001. 
(a)

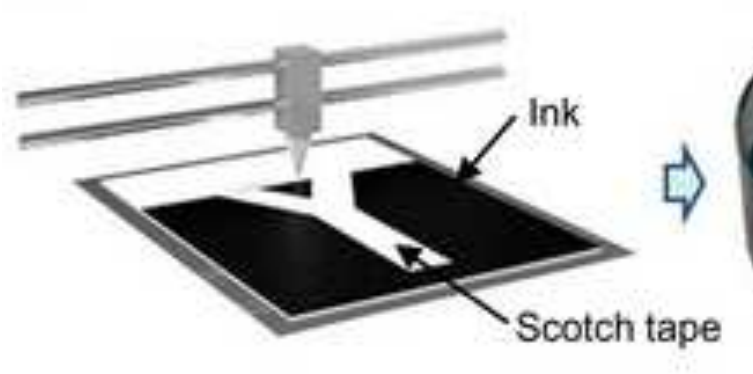

Negative pattern

(f)

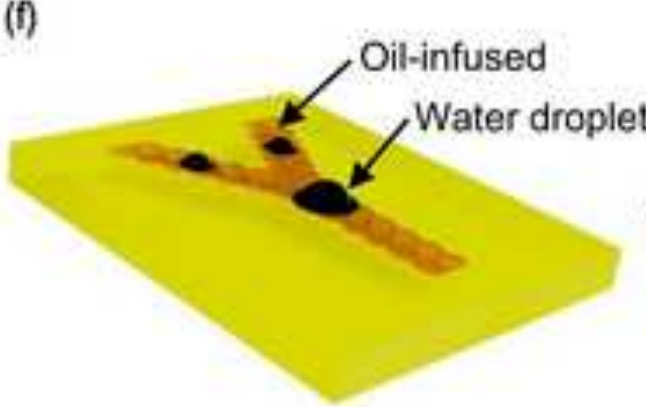

Ultra-slippery PDMS surface (b)

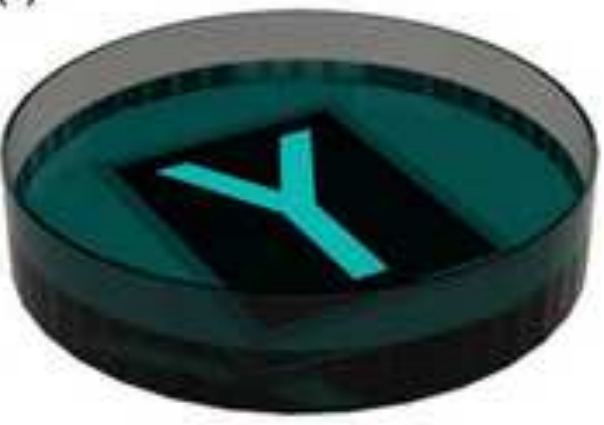

Immerse in water

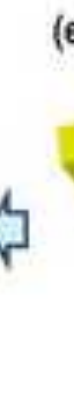

(e)

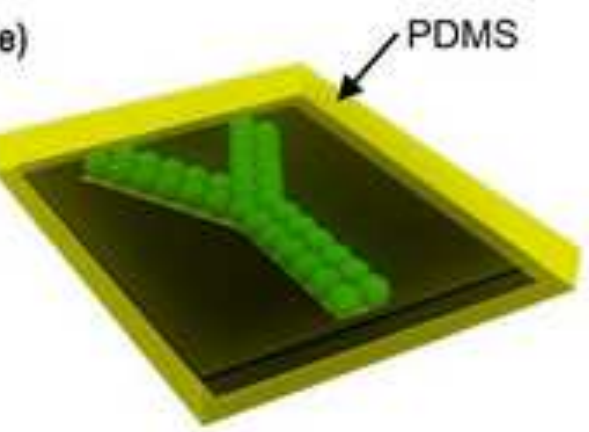

PDMS casting
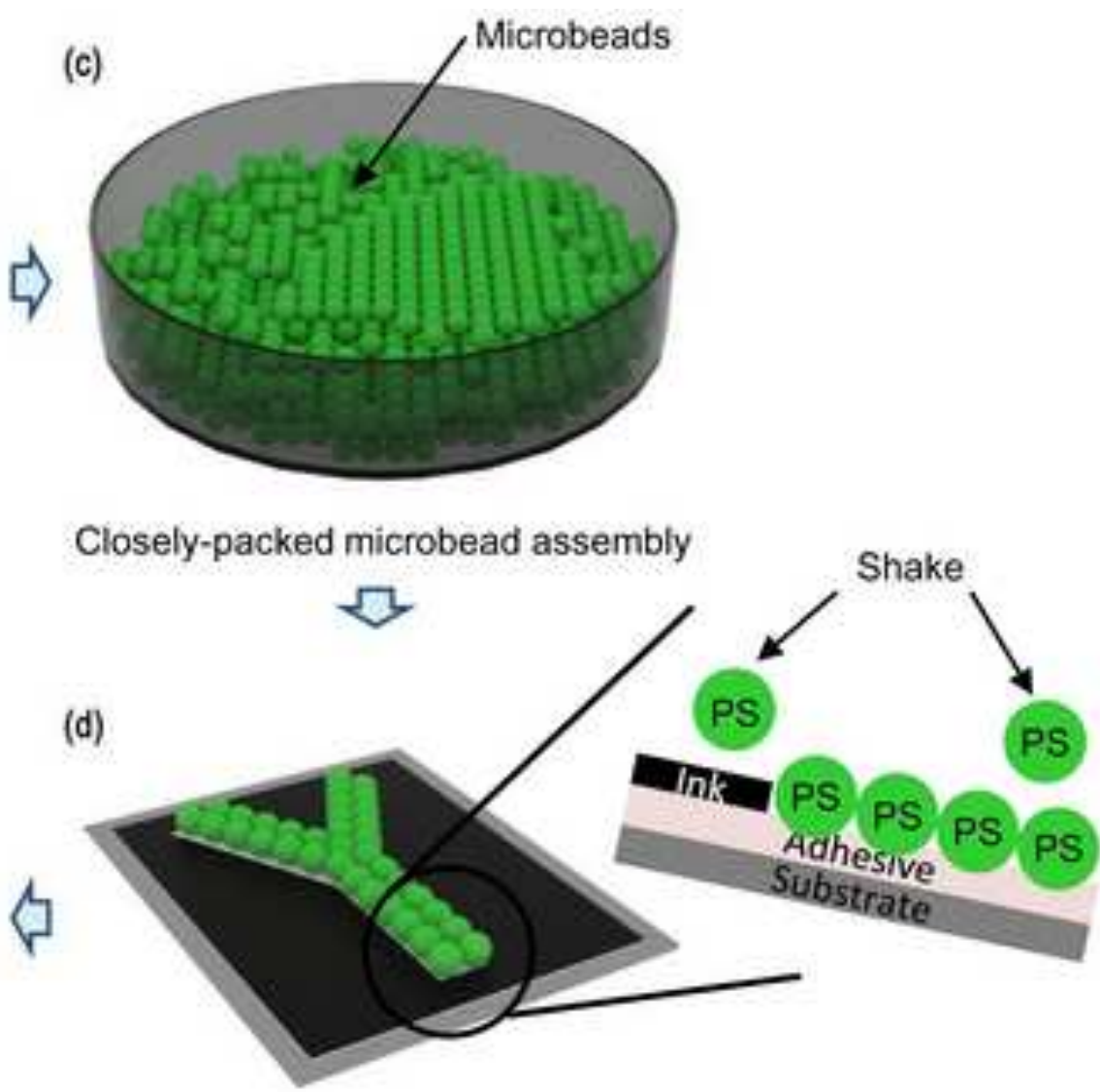

Monolayer microbeads 

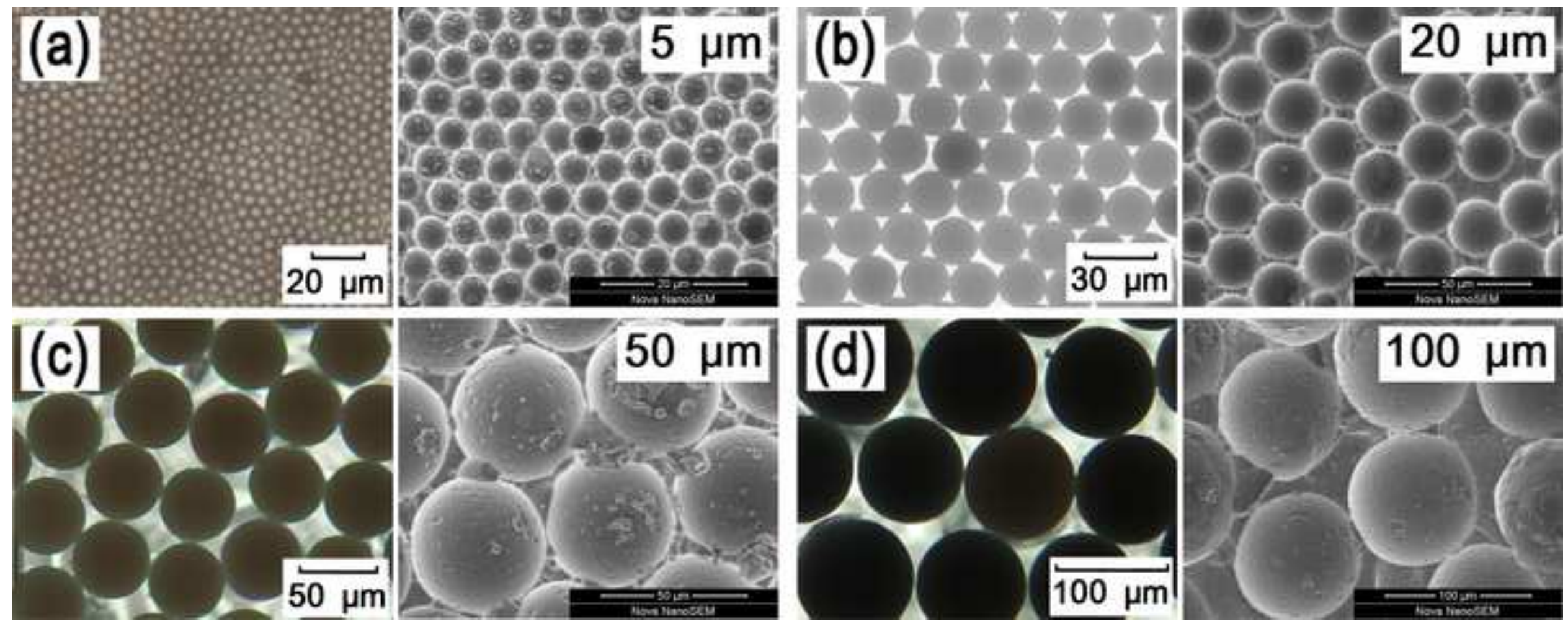

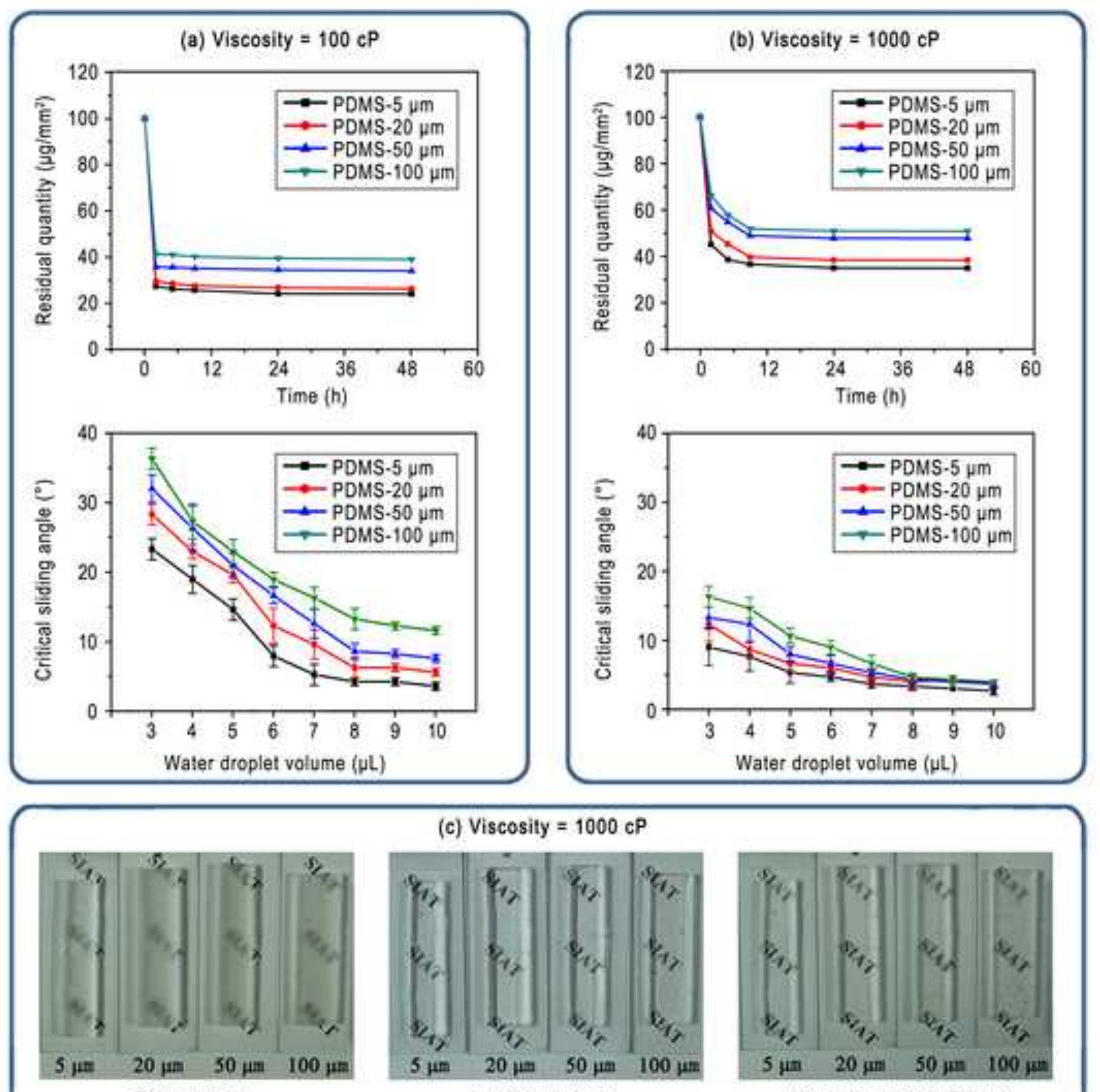

(c) Viscosity $=1000 \mathrm{cP}$

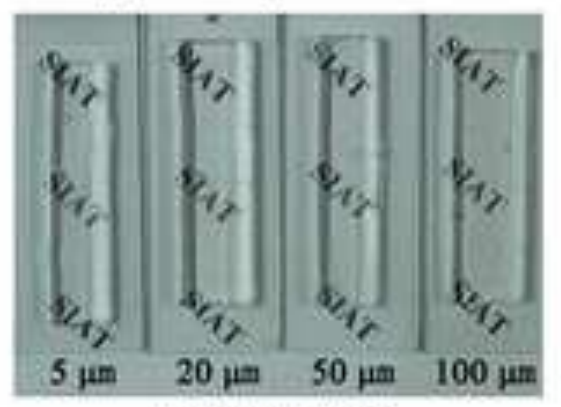

Lubricant infused

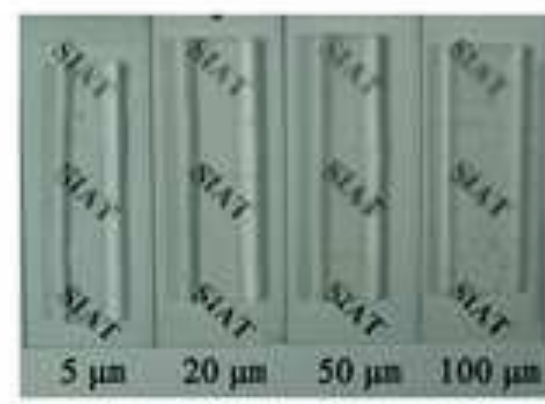

Vertically placed 48 hours 


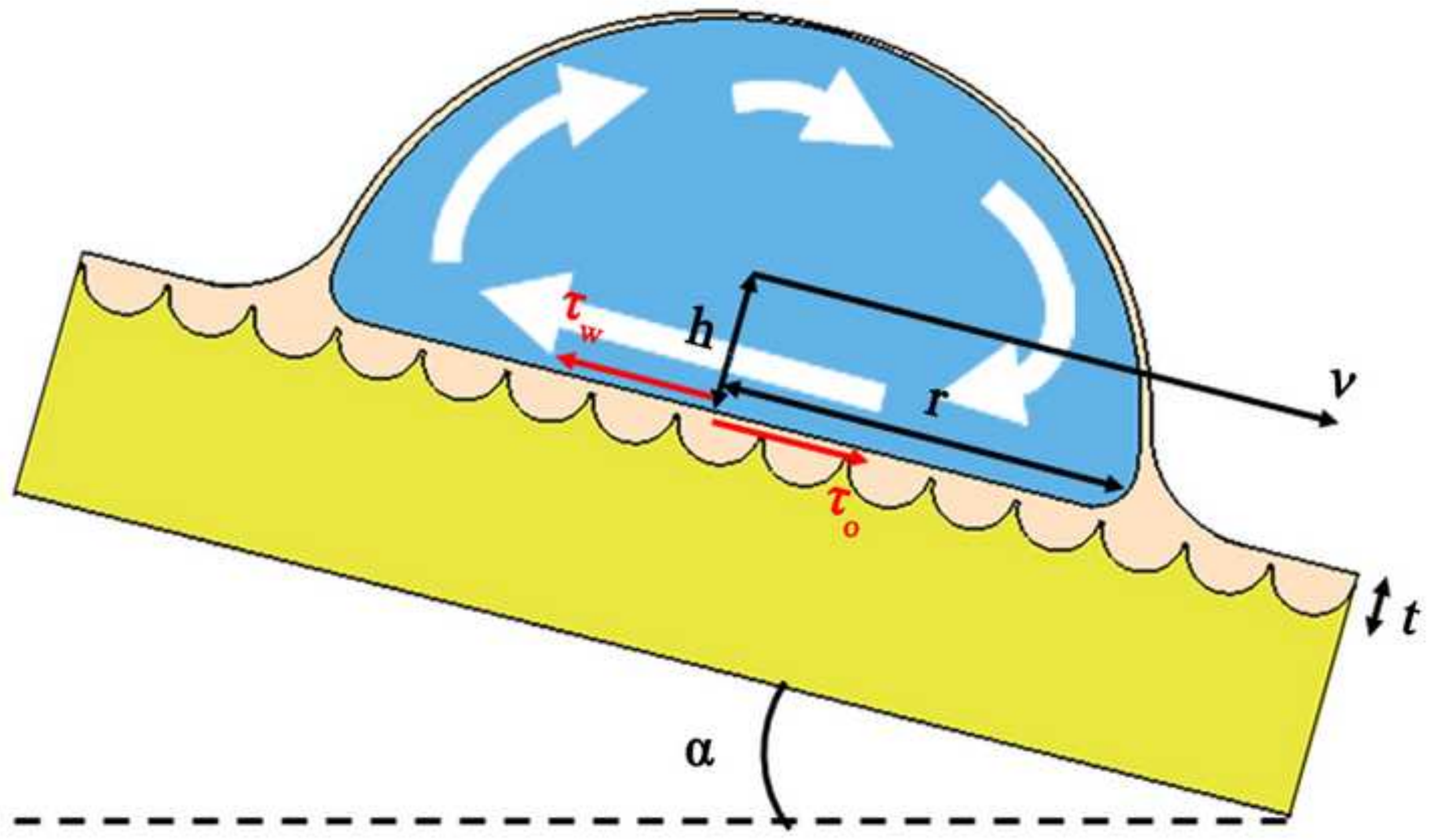




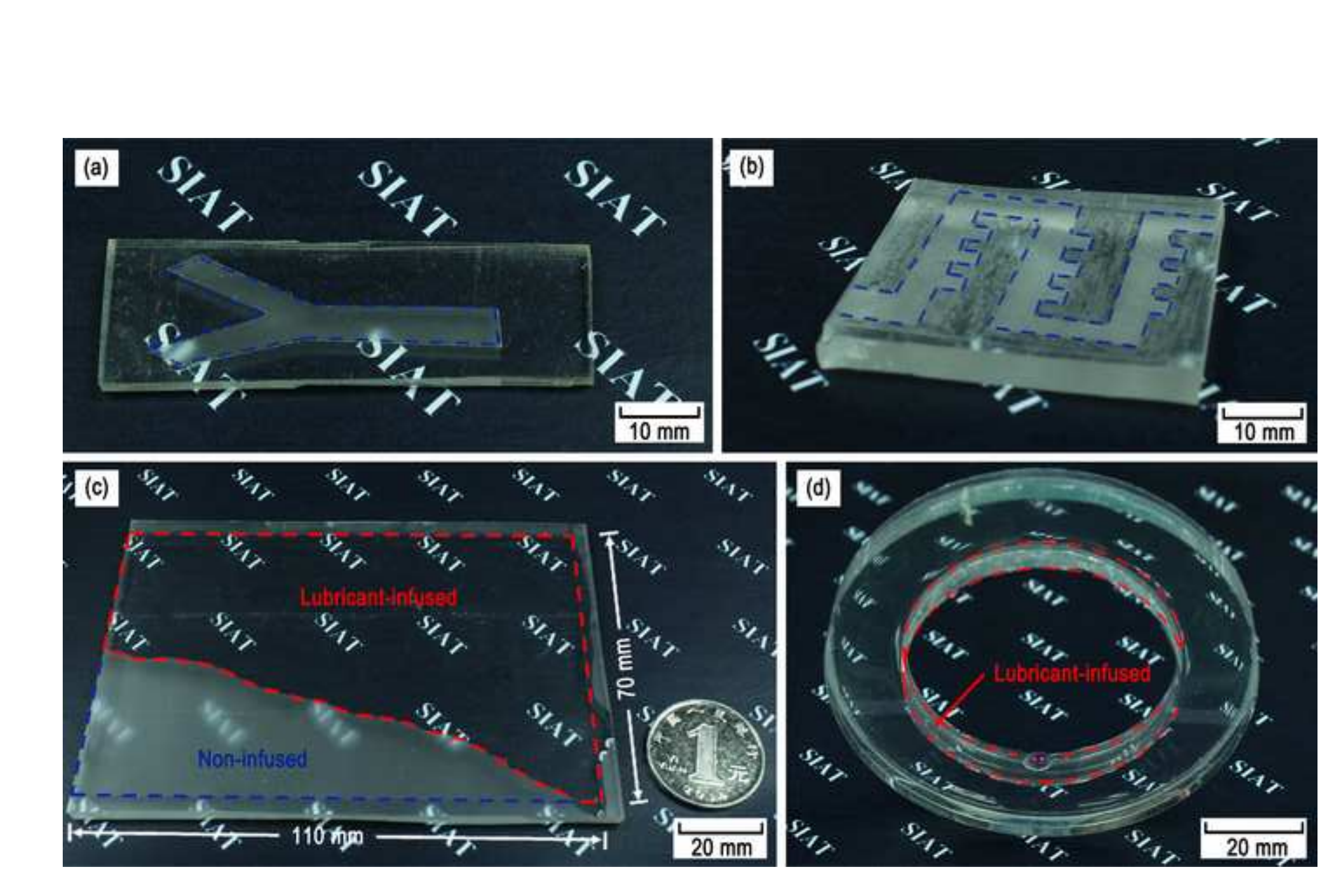

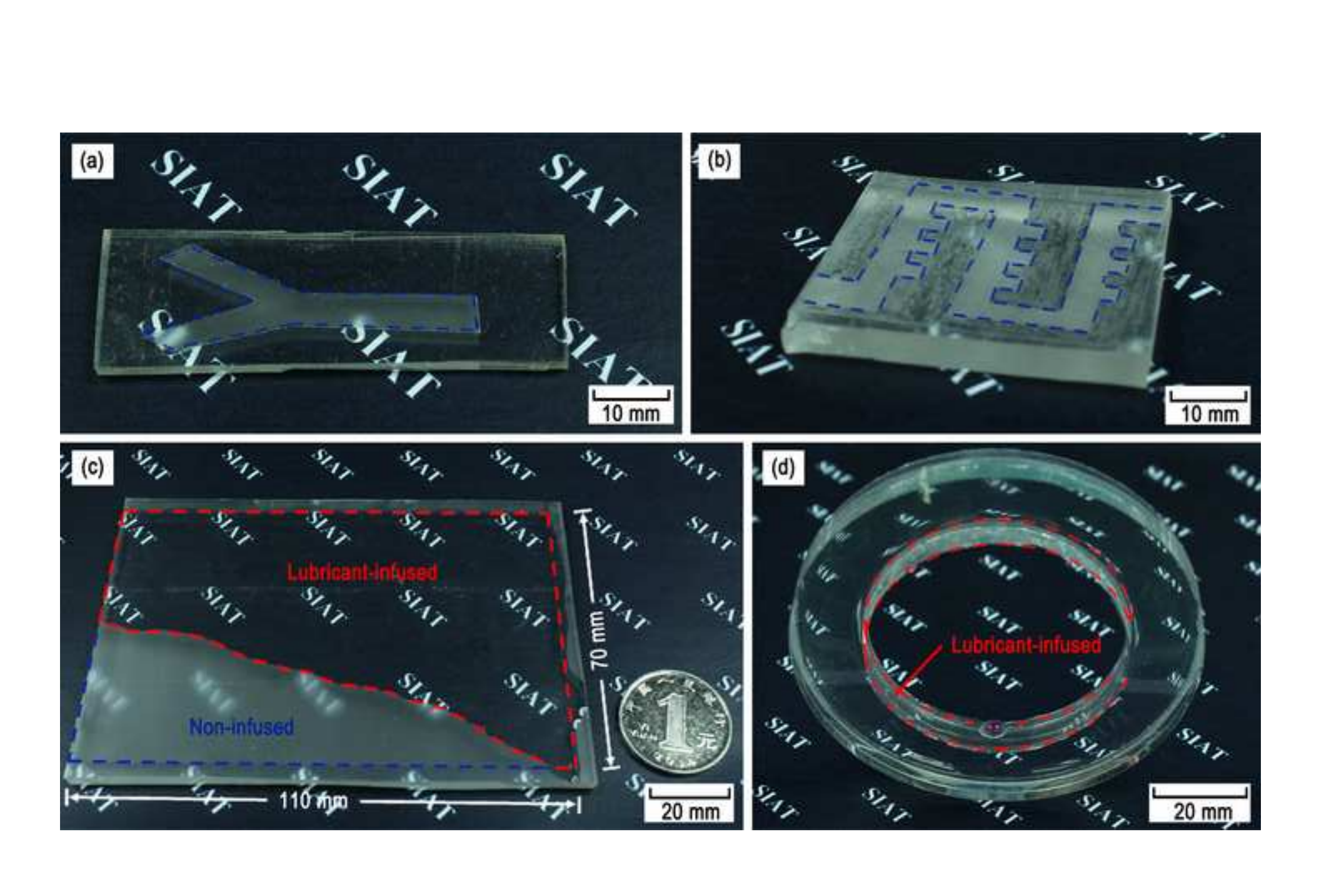$$
\text { s. }
$$

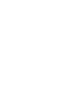$$
\text { ( }
$$$$
\text { ( } 1
$$
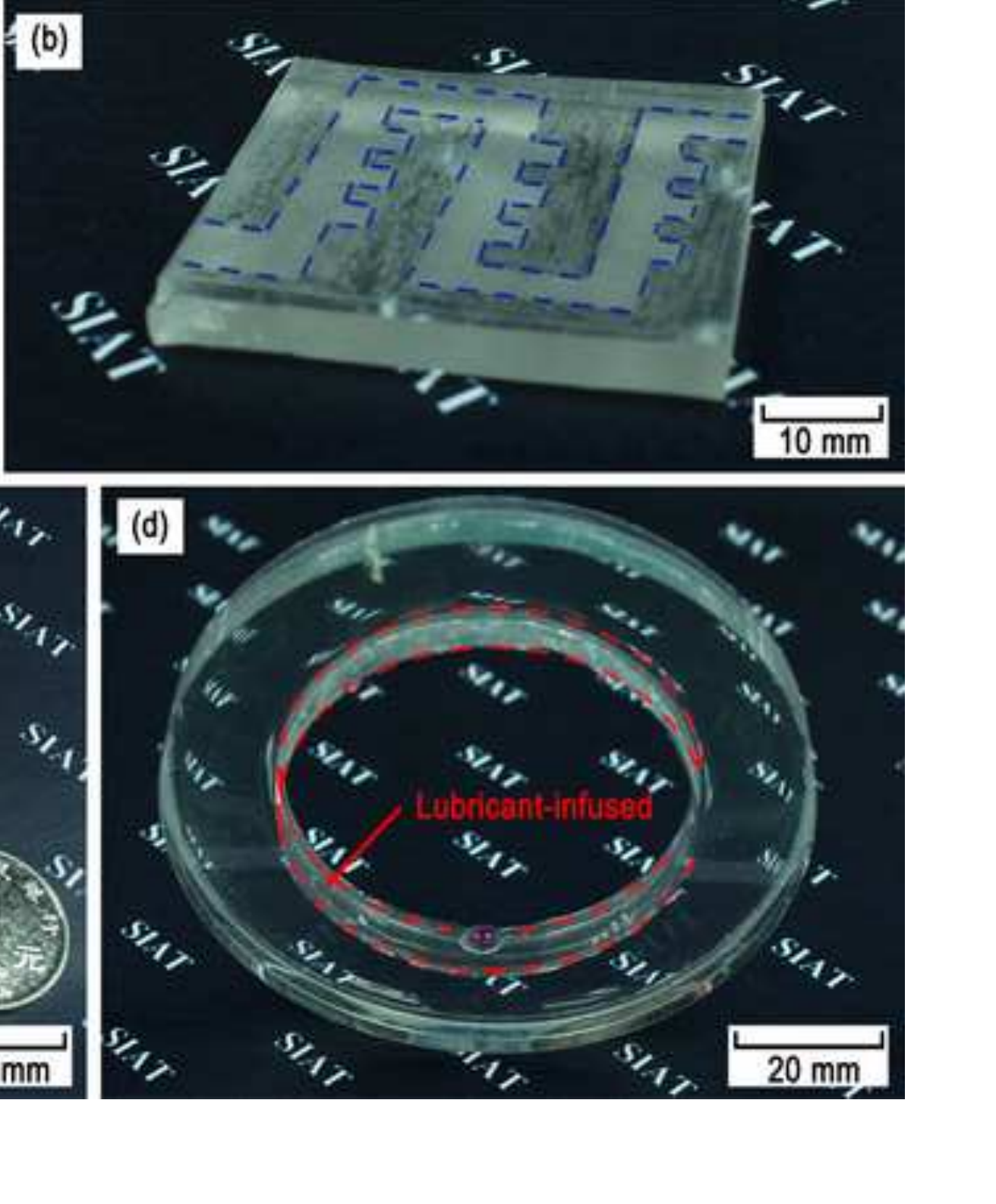

(

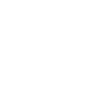

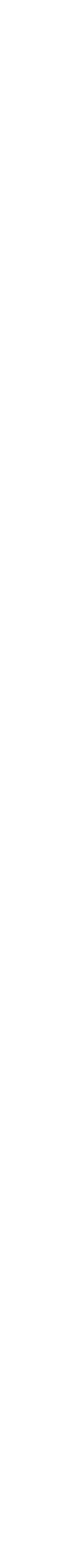

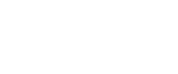

$$
\mathrm{de}
$$$$
-1
$$$$
\text { 象 }
$$
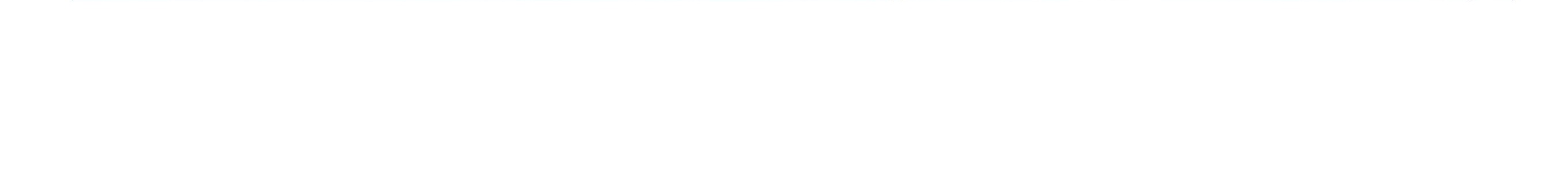

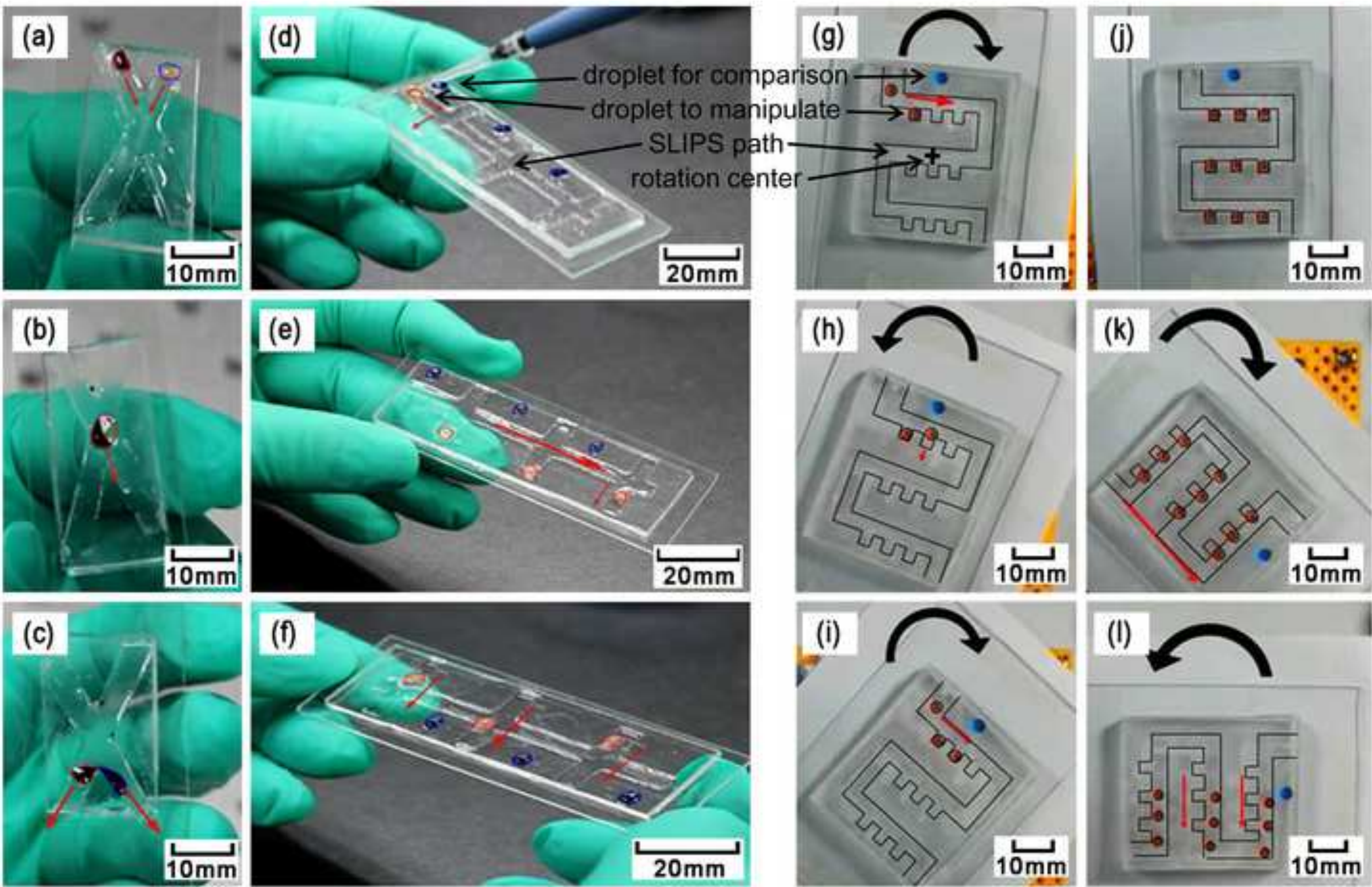
(a)

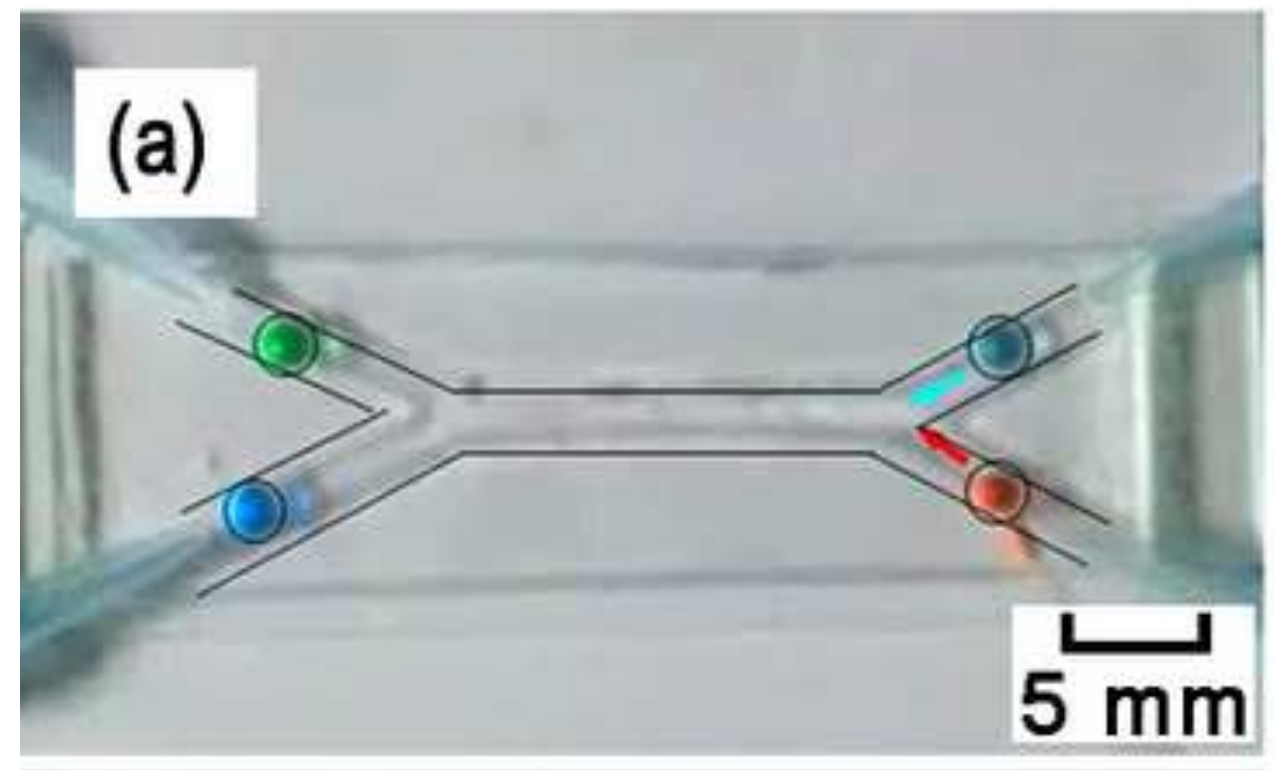

(c)

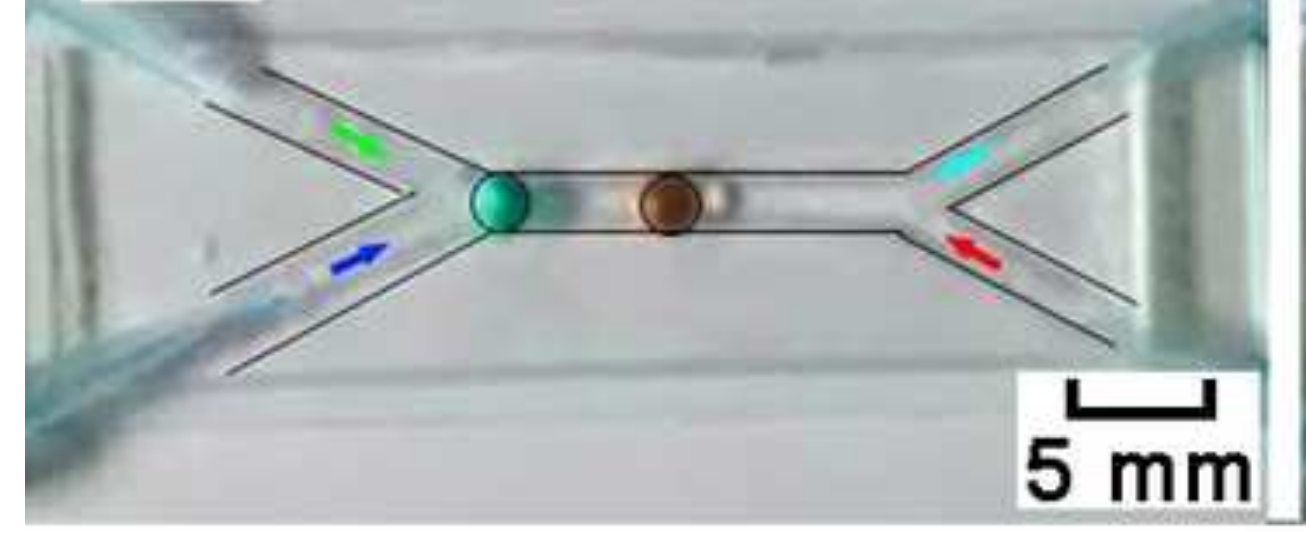

(b)

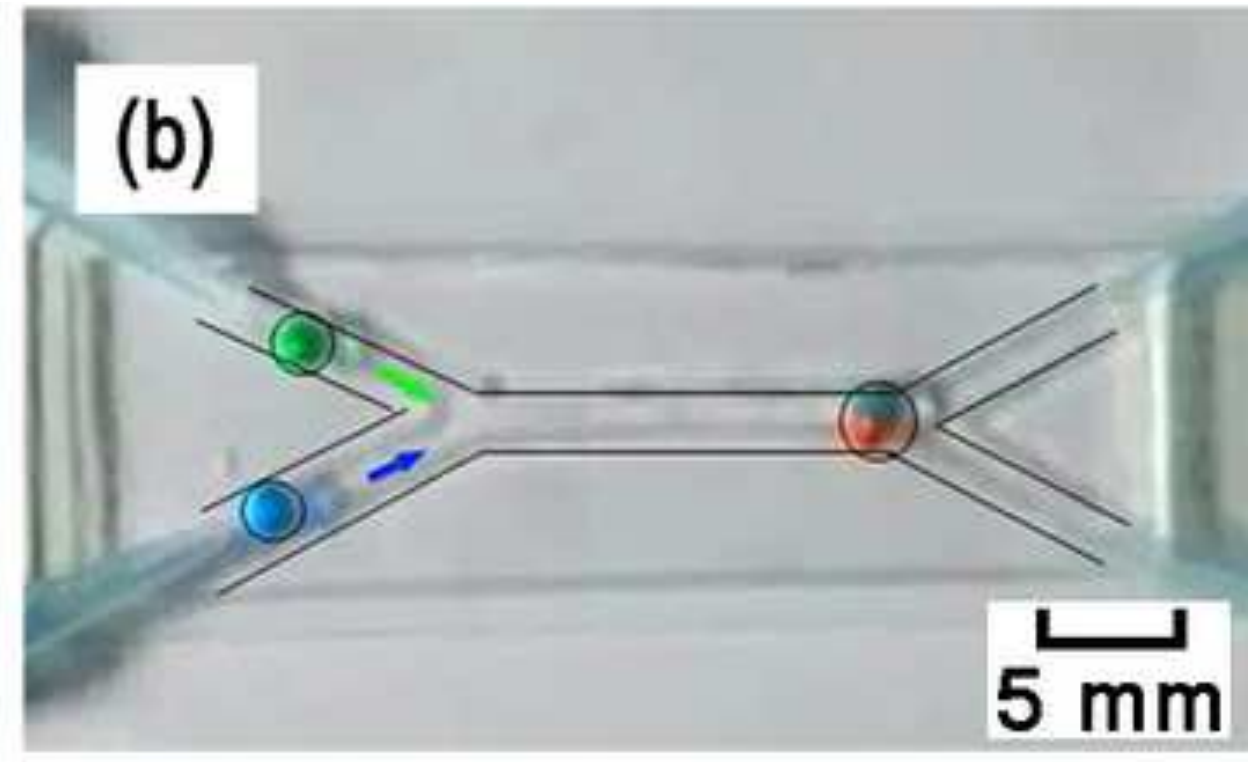

(d)

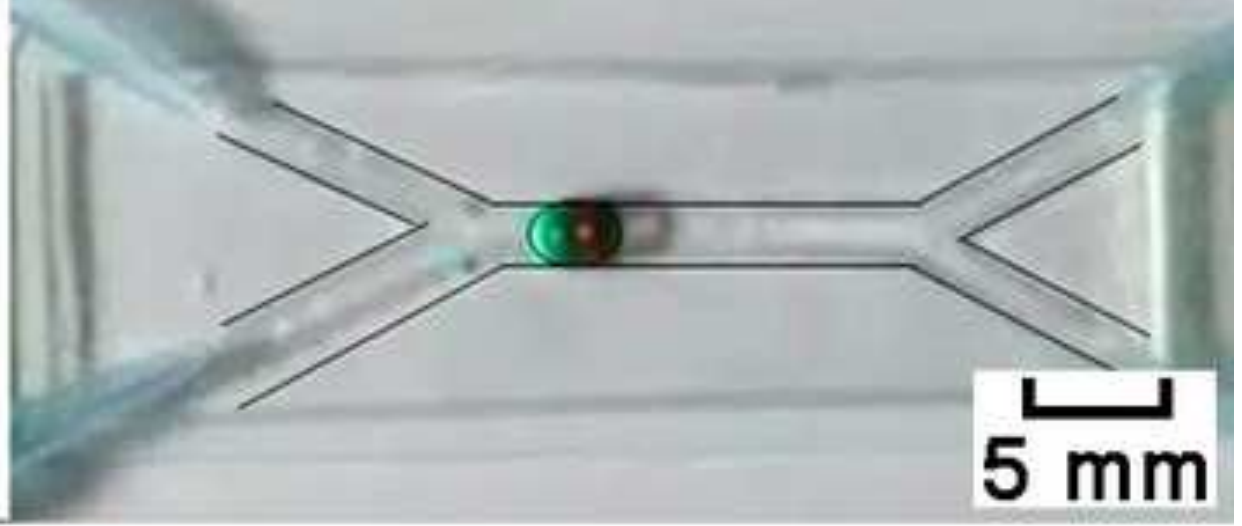




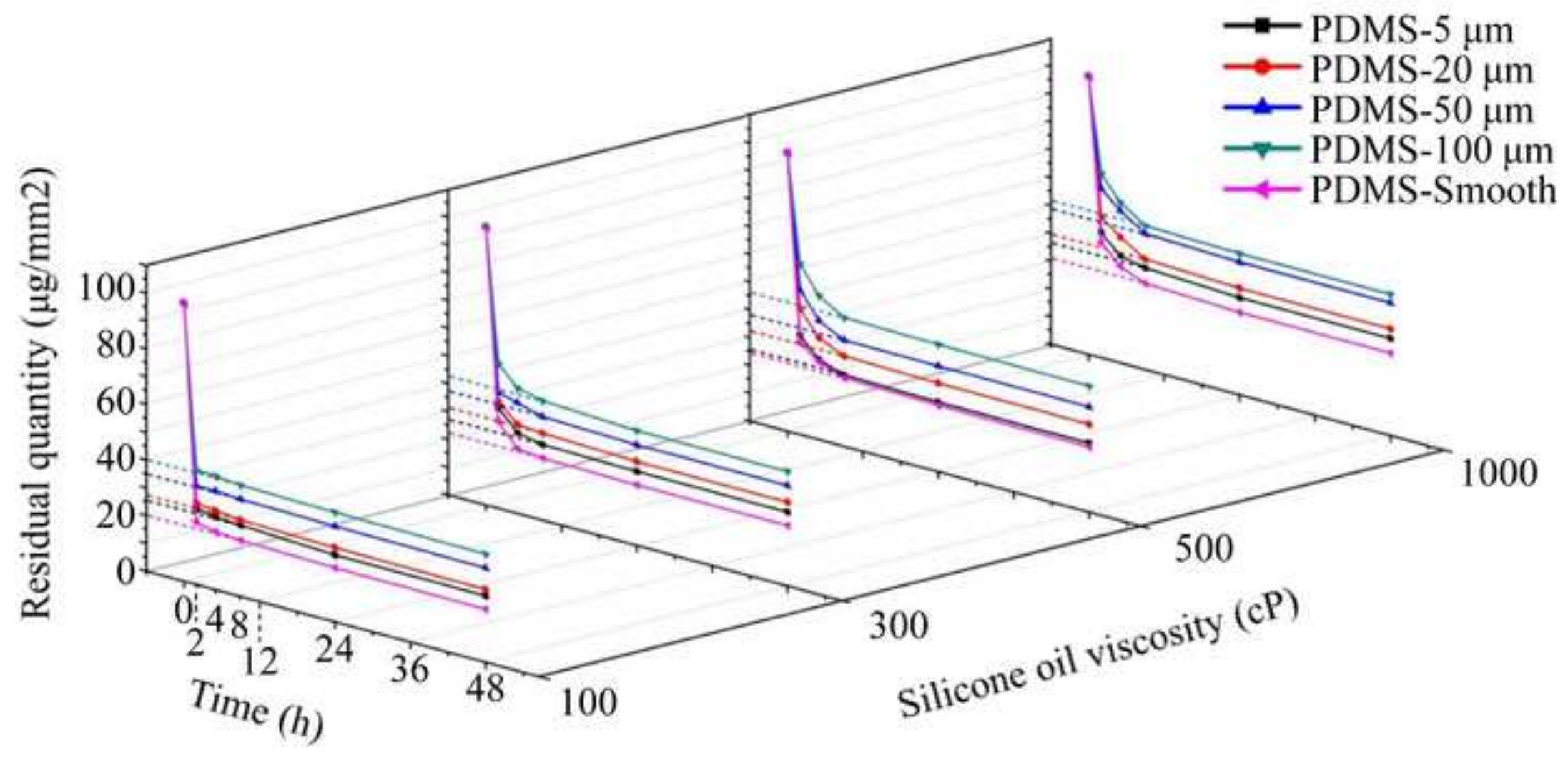




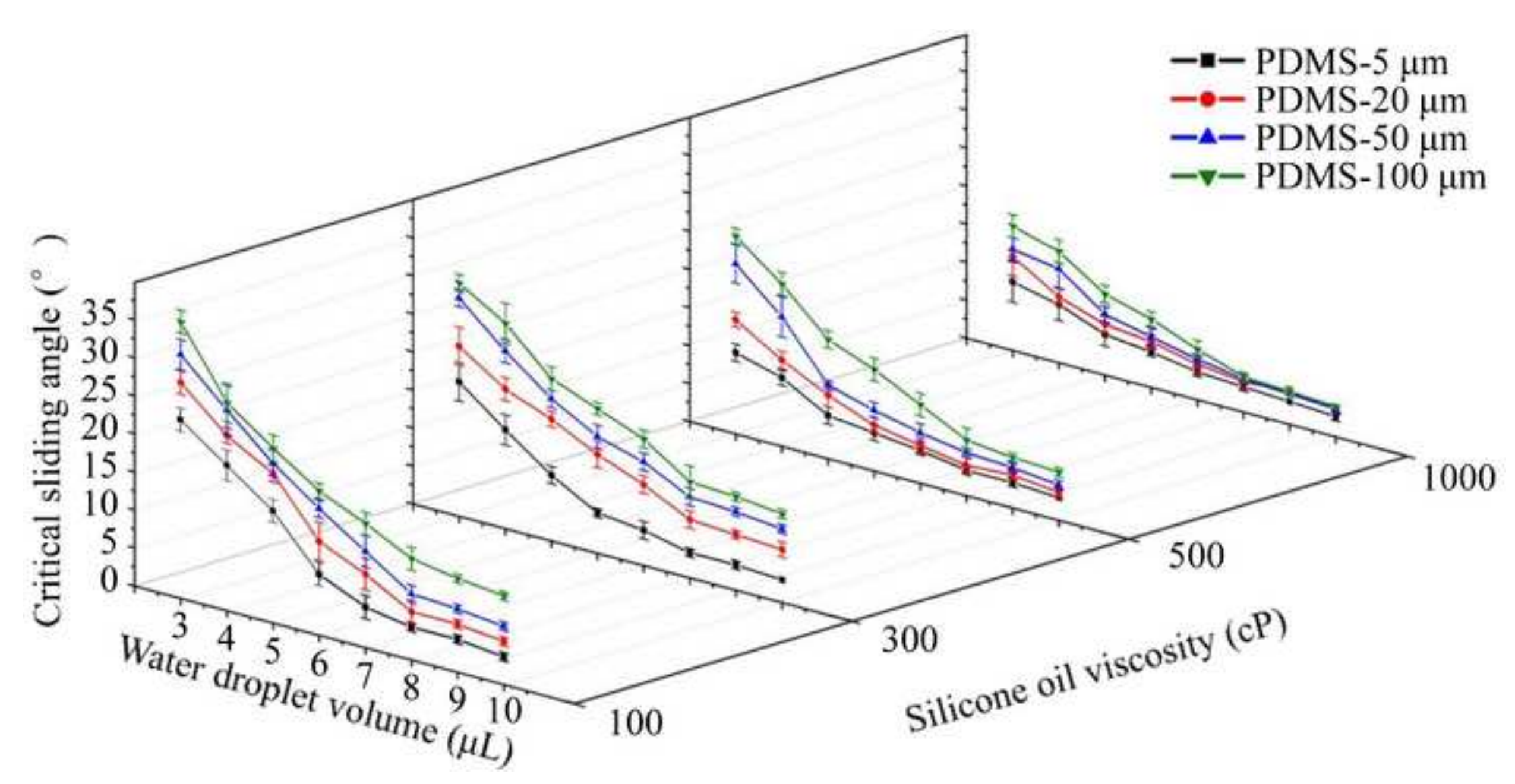

